\title{
EL PROCESO DE INFORMALIZACIÓN \\ EN EL DESARROLLO DEL RUNNING
}

\author{
Raúl Sánchez García \\ raul.sanchezg@upm.es \\ Universidad Politécnica de Madrid
}

\section{RESUMEN}

El objetivo de este artículo consiste en aplicar la teoría de la informalización de Cas Wouters -que amplía las teorías sobre el proceso de civilización de Norbert Elias- al análisis de la actividad deportiva del running tal y como se desarrolló desde la segunda mitad del s. $x x$ en EE. UU. y desde mediados de los años 2000 como tendencia deportiva global. Como parte de la metodología, se han analizado fuentes escritas más relevantes tales como la revista Runner's World, libros de referencia, así como foros de discusión referidos a las actividades propuestas. Como conclusión principal podemos decir que el desarrollo del running se identifica con un patrón informalizador en espiral, pasando por diversas fases de informalización y reformalización durante las cuales hubo una variación de los distintos tipos de valores y discursos asociados a la actividad.

Palabras clave: informalización, Wouters, Elias, running, carrera de obstáculos, carrera descalzo/mínima.

\author{
THE INFORMALISATION PROCESS IN \\ THE DEVELOPMENT OF RUNNING
}

\section{Abstract}

This paper applies Cas Wouter's informalisation theory -which continues Norbert Elias's theory on the civilisation process - to the development of running as it unfolded since the second half of the twentieth century in USA and since the mid 2000s as a global sport trend. Different written sources such as the magazine Runner's World and other relevant books and blogs have been used for the analysis. The development of running followed an spiral informalising pattern, alternating between phases of informalisation and reformalisation during wich the discourses and values attached to the activity changed.

KEYwORDs: informalisation, Wouters, Elias, running, obstacle course race, barefoot/minimal running. 


\section{INTRODUCCIÓN}

La presente investigación aplica al análisis del desarrollo deportivo -más en concreto al running ${ }^{1}$ - la teoría de la informalización, desarrollada por Cas Wouters desde los años 70 (Wouters 1976, 1986, 1999, 2004, 2007, 2014, Wouters y Dunning, 2019), continuando los análisis sobre el proceso de civilización que llevó a cabo Norbert Elias (1995[1939)]. Según Wouters, Elias solo había identificado la tendencia formalizadora de tal proceso que había sido predominante desde finales de la Edad Media hasta la última parte del s. $\mathrm{XIX}^{2}$. Desde ese momento, Wouters consideraba que la tendencia informalizadora ganó predominancia de forma general ${ }^{3}$. Gracias a un estudio procesual de más largo alcance que comparaba los casos de EE.UU., Alemania, Inglaterra y Holanda tanto en lo que se refería a los modales (psicogénesis) como a procesos sociales más generales (sociogénesis), Wouters identificó tres momentos clave en el impulso informalizador: último tercio del s. XIx, la década de los años 20 y la revolución cultural de los 60 y 70. Durante períodos que Wouters denominaba de reformalización, los códigos informalizados de la etapa anterior se integraban y pasaban a formar parte de los estándares establecidos en la sociedad.

Originalmente, Wouters desarrolló su teoría a partir de la observación directa de una aplicación más flexible de códigos, normas y modales en la Holanda de las décadas de 1960 y 1970. Al contrario de los discursos representantes del «pánico social» de la época que preconizaban el caos o la destrucción de la sociedad, para Wouters tales comportamientos implicaban un desarrollo más complejo de las tendencias civilizadoras. La mayor integración entre distintos grupos sociales debidos a la clase, al género, la etnia y las generaciones en un nivel sociogenético deman-

${ }^{1}$ He decidido utilizar el término proveniente del inglés «running» para designar la práctica deportiva de carrera a pie. Además de «running» se utilizaron, tanto en los países anglosajones como en otros, términos tales como «jogging». En determinados momentos se hará alusión a los términos específicos, ya que contienen matices importantes.

${ }_{2}$ Norbert Elias también ańadió interesantes conceptos relativos a la informalización. El autor desarrolló el «gradiente sincrónico de formalidad e informalidad» (en un momento dado), el "gradiente diacrónico de informalización» (como proceso histórico) y el «tramo formalidad-informalidad de una sociedad» (Elias, 1996: 28-29). Este último trataba la diferencia en los modales y comportamientos entre los encuentros sociales públicos, pactados y ritualizados (formalizados), dependientes en mayor medida de las costumbres de las clases altas y los comportamientos más naturales o relajados (informales) de la vida privada, que presentan una mayor identificación con las clases bajas. Cuanto más empinado sea el tramo, menos civilizada será una sociedad (más jerarquizada, menor identificación entre grupos sociales). Cuanto menos empinado sea el tramo, más civilizada una sociedad (menor jerarquía, mayor identificación entre grupos sociales). Sin embargo, existen matices importantes entre la concepción de Elias y Wouters sobre la informalización. Ello se originaba a partir del distinto punto de partida de ambos; mientras que Elias mantenía el foco en el primer término de su famoso binomio "disminución de contrastes, aumento de variedades» (Elias 1995 [1939]), Wouters (1976) se centraba en el segundo.

${ }_{3}$ No obstante, predominancia no implica exclusividad; de igual modo que Elias siempre hablaba del cambiante equilibrio entre tendencias civilizadoras y descivilizadoras, Wouters hablará de un equilibrio entre tendencias formalizadoras e informalizadoras (Sánchez García, 2017). 
daba la aplicación de comportamientos más flexibles, más moderados y controlados en un nivel psicogenético.

Por tanto, si bien desde la teoría general elisiana las tendencias informalizadoras podrían tener apariencia de descivilización, en verdad implican un mayor grado de civilización: mantener el control de uno mismo en una mayor variedad de situaciones sociales que demandan una mayor adaptabilidad. Wouters explica cómo en el nivel psicogenético, la informalización implica el desarrollo de un habi$t u s^{4}$ de tercera naturaleza (Wouters, 2007: 212), donde la vida emocional y afectiva estaba más presente a la propia consciencia de los sujetos. Al contrario del habitus como segunda naturaleza del que hablaba Elias por el cual, durante el proceso de civilización, los controles externos se habían ido internalizando en el propio sujeto hasta convertirse en un modo de autocontrol más o menos automático, el habitus de tercera naturaleza implica una mayor reflexividad vinculada a la emancipación de las emociones, las cuales se viven de forma más consciente sin el miedo a perder el control sobre ellas. Según Wouters (2004: 208), el avance era desde la conciencia (mayor dirección desde el superego, predominante en fases de formalización) a la consciencia (mayor dirección desde el ego, predominante en fases de informalización).

La teoría de la informalización de Wouters está íntimamente relacionada con el ámbito del ocio y del deporte tal y como lo concebían Elias y Dunning (1992). Estos autores veían el ocio y el deporte como inventos sociotécnicos en los que se permitía la vivencia de estimulación emocional dentro de cierta seguridad; esas actividades permitían un "controlado descontrol de los controles emocionales», expresión central en la propia teoría de Wouters para hablar de la informalización (Wouters, 2007: 212). De hecho, las ideas de Wouters pueden ayudar a realizar una relectura de la teoría de la búsqueda de la emoción en el ocio y el deporte. Mientras que Elias y Dunning consideraban el ocio y el deporte como una especie de mecanismo de compensación en las sociedades occidentales de la segunda mitad de s. Xx, con alto grado de civilización, altamente rutinizadas y poco estimulantes, Wouters consideraba el desarrollo del ocio y el deporte en esas sociedades, no como mecanismo de compensación, sino como síntoma de tendencias generales (informalizadoras) que se estaban dando a la vez en otros ámbitos sociales del momento. Por tanto, Wouters cogió la expresión elisiana del «controlado descontrol de las restricciones emocionales» del contexto limitado del ocio y el deporte y la utilizó para señalar una

${ }^{4}$ Si bien este término ha sido asociado en la literatura sociológica a Pierre Bourdieu (1991), el propio Norbert Elias hizo un uso anterior del término, con sus propias peculiaridades (véase Sánchez García, 2008 para una comparación del concepto en ambos autores). En el caso de Elias, (1995: 453-455, 463, 488, 491, 498, 179, 526; 1996: 15) el habitus debe entenderse fundamentalmente dentro del marco de la teoría del proceso de civilización. Se refiere al tipo de economía afectiva (Affekthaushalt) o modelación de los impulsos (Triebmodellierung), resultante de la relación concreta dentro de los individuos entre la espontaneidad afectiva del ello y el grado y tipo de constricciones, las cuales pueden ser tanto externas (debidas a la autoridad que otros ejercen sobre el individuo) como internas (debidas al superego en el sentido freudiano). 
tendencia general de los procesos sociales y psíquicos en las sociedades occidentales del s. xx (Wouters, 2007: 240).

Pero aquí no acaba la posible relación entre las teorías de Wouters y el ámbito deportivo. Como trata de mostrar el presente artículo, la teoría sobre la informalización tiene una relevancia fundamental para entender el desarrollo del propio fenómeno deportivo moderno. Hasta el momento se han realizado algunos estudios que vinculan informalización y deporte. Por ejemplo, se han identificado patrones informalizadores en el desarrollo de la esgrima en Francia durante el último tercio del s. XIX (Sanchez García, 2018a); en el desarrollo de las artes marciales en Japón durante la década de 1870 , el período de entreguerras y la segunda mitad del s. xx (Sánchez García, 2018c); en el desarrollo internacional de las artes marciales mixtas (MMA) durante la segunda mitad del s. Xx (Sánchez García y Malcolm, 2010; Sánchez García, 2018b). La presente investigación pretende continuar esta línea de análisis, aplicando en este caso la teoría de la informalización al desarrollo del running tal y como se desarrolló desde la segunda mitad del s. xx en EE. UU. y desde mediados de los ańos 2000 como tendencia deportiva global.

\section{OBJETIVOS Y METODOLOGÍA}

El objetivo principal de este trabajo consiste en identificar y relacionar cambios que ocurren en la sociedad en general con cambios en los valores vinculados a la actividad del running en el período que va desde la década de 1960 hasta la actualidad.

Los datos utilizados para observar los cambios en los valores vinculados a esa actividad provienen de los libros más relevantes en cada época y de las publicaciones periódicas de referencia en ese ámbito; por ejemplo, la revista Runner's World, que no ha parado de publicarse de forma ininterrumpida desde medidados de los 60, cuando se llamaba Distance Running News.

\section{RESULTADOS Y DISCUSIÓN}

LA FASE DE FORMALIZACIÓN/INFORMALIZACIÓN EN EE.UU. DURANTE LOS AÑOS 60 Y 70

Durante las décadas de 1960 y 1970 se produjo en EE.UU. una mayor integración social y un uso más variado y flexible de costumbres y códigos sociales. La nivelación de grupos étnicos y de género y la expresión de sentimientos antisuperioristas devinieron prominentes a través del activismo social, como, por ejemplo, en la segunda ola del feminismo o el movimiento por los derechos civiles. La generación del Baby-Boom experimentó una revolución contracultural, ejemplificada por jóvenes de clase media que trataban de rechazar los valores tradicionales, descolgándose del sistema y creando nuevos modos de vida tales como los ejemplificados por el movimiento hippie. Este cambio también se dejó notar en el ámbito deportivo. Durante los años 60 y 70 se produjo la emergencia de nuevas formas de 
deporte: los deportes alternativos (Rinehart, 2000); deportes whiz (Midol, 1993); o como más recientemente los denomina Wheaton (2004), deportes como estilo de vida. Los valores contraculturales asociados a esas prácticas estaban fuertemente influidos por los llamados deportes californianos (Pociello, 1979, 1995; Loret, 1995), ya que la costa oeste de los EE. UU. era uno de los epicentros de ese cambio hacia valores alternativos en lo cultural. Actividades tales como el surf, el skate, la escalada, el BMX, etc., representaban un giro informalizador en lo deportivo, con un enfoque anticompetitivo, contra las reglas, rechazando la mentalidad de ganadores y perdedores (lo que Wouters [2007] denominada superiorismo). En lugar de la competición contra otros, el centro lo ocupa la exploración y crecimiento personal.

En el ámbito del running tales cambios en los valores también ocurrieron, sobre todo en los años 70, cuando la actividad de carrera a pie en EE. UU. llegó al culmen de participación ${ }^{5}$. No obstante, sobre todo durante la década de los 60 , tendencias formalizadoras siguieron teniendo predominancia, conectando la carrera a prácticas de salud y de responsabilidad individual, asociada a profesionales médicos y a entrenadores como Lydiard y Bowerman, con una formación proveniente de la etapa previa a la guerra basada en la ética del trabajo, el esfuerzo y la heroicidad. La carrera (en estos momentos denominada como jogging) era parte de programas de fitness relacionado con la salud, orientada principalmente hacia aquellos de mediana edad en una sociedad preocupada por el envejecimiento. También mostraba una relación con la estética, ya que, como apuntaba Bowerman, ayudaba a tener un mejor aspecto, a perder peso y disminuir la cintura, aumentando la autoconfianza y la capacidad sexual. Más aún, el complejo ejercicio-salud-estética presentaba un trasfondo moralista: estar fuera de forma era un síntoma de irresponsalibilidad. Bowerman y Harris afirmaban que «la persona gorda feliz es un mito. Normalmente se encuentra fatal, avergonzado de su apariencia». (1967: 21).

Este tipo de valores se encarnaban en el propio diseño de las zapatillas, que se centraba en la dureza, la durabilidad y presentaba un sistema ligero de amortiguación.

Durante los años 70 vemos cómo la tendencia informalizadora ganó ascendencia y un tono contracultural, basado en el antiautoritarismo, diversión y un retorno a formas más naturales de correr, se dejó ver en las obras de referencia de una segunda generación de entrenadores tales como George Sheehan, Mike Spino o James Fixx. La carrera ahora se enfocaba desde una posición antimédica, favoreciendo programas holísticos y de autoayuda que demandaban una responsabilidad personal. Durante esta fase el término jogging empezó a coexistir con el de running. El corredor (runner) expresaba un estilo de vida integral, más allá de la limitada relación de la carrera con la salud. Lo central en lo corporal era la parte interna, de conocimiento personal, no la cuestión externa de la forma estética. La carrera se definía desde lo personal y uno podía definirse como corredor legítimo aunque no estuviera entre la élite del deporte. Como expresaba Fixx:

${ }^{5}$ De acuerdo a una encuesta nacional de 1979, 30 millones de estadounidenses realizaban actividades de carrera (Henderson, 1983: 347). 
Para muchos de nosotros, la carrera es un regalo especial, una oportunidad para competir primordialmente contra uno mismo y ver cuánto más rápido podemos ir respecto a cómo lo hacíamos antes [...]. Es por eso que hay tanta camaradería en las carreras. Otros corredores no son tus competidores; tú lo eres (1977: 178).

El rechazo de los sentimientos de superioridad estaba presente en el modo en el que el autor definía la carrera como «probablemente la más democrática. Los corredores carecen casi por completo de discriminación por raza, sexo, edad o clase» (Fixx, 1977: 38). Además, su consideración de la actividad mostraba el anhelo ecológico de la vuelta a un pasado no urbano:

Como corredores creo que nos conectamos con la cadena interminable de la historia. Experimentamos lo que habríamos experimentado diez mil años atrás, comiendo frutas, frutos secos y vegetales y manteniendo nuestros corazones y pulmones en forma mediante el constante movimiento (Fixx 1977: 249).

El contraste respecto a la etapa anterior también se dejó notar en el diseño de las zapatillas. Reflejando la tendencia de los nuevos valores asociados a la carrera, lo fundamental en las nuevas zapatillas era la ligereza y la flexibilidad del antepié.

\section{REFORMALIZACIÓN TEMPRANA EN LA DÉCADA DE LOS 80}

Alrededor de 1980 se produjo una vuelta al conservadurismo con el neoliberalismo en la era Reagan, con énfasis especial en la austeridad fiscal, el libre mercado y una falta de intervención gubernamental. Los valores familiares basados en la moralidad, en la propiedad y la decencia eran aclamados. De modo general, sentimientos de superioridad y competición eran potenciados de nuevo. Durante esta década, marcada por la tensión de la guerra fría, el héroe de acción encarnado en Sylvester Stallone y Arnold Schwarzenegger, muestra un físico muscular desproporcionado en títulos tan conocidos como Rocky, Rambo, Terminator, Conan, o Comando (Dutton, 1995).

La generación más joven desarrolló una cultura del consumo basada en la ostentación de la imagen personal: el narcisista culto al cuerpo como objeto de deseo que debía ser disciplinado a través de la dieta y el ejercicio y como modo de expresión de juventud, vitalidad, energía y movilidad. El yuppie representaba la máxima figura del éxito en un tiempo en el que la emancipación colectiva de las décadas previas desaparecía para dar paso a un tipo de liberación basada únicamente en la libertad personal. Las actividades físicas se vieron influidas por esa reformalización y se convirtieron en mecanismos explícitos para conseguir la imagen corporal deseada. Como expresaban Kirk y Coulquhoun (1989), el término fitness contenía al mismo tiempo nociones de salud y apariencia estética; lo importante no era solo estar sano, sino también (sobre todo) parecerlo porque el cuerpo saludable era atractivo. Como decía un artículo del USA Today de la época: "Creemos que tener un cuerpo adecuado significa tener nuestras vidas bajo control. El cuerpo Nautilus 
[máquina de fitness de la época], sin grasa, sin humo, se ha convertido en un símbolo de estatus. Tener uno de esos es tan bueno como tener un BMW» (14 de septiembre, citado en Howell, 1991: 263). Featherstone (1987: 126) apuntaba que esa tendencia era especialmente marcada entre las clases medias, las cuales eran asiduas practicantes de la carrera, el aerobic o las actividades al aire libre como forma de dar respuesta a su preocupación con la forma de presencia personal. De acuerdo con Ingham (1985), la atención sobre lo corporal aumentó gracias a la política gubernamental de no intervención en materia de salud, favoreciendo la toma de responsabilidad individual dentro de la elección personal de estilos de vida. El cuidado del cuerpo se convirtió en una disposición moral; los malos hábitos eran responsables del cáncer, del sida o de los ataques de corazón. Estar enfermo, envejecer, presentar una falta de forma o tener sobrepeso eran marcadores de degeneración moral de aquellos que no podían responsabilizarse de ellos mismos y por tanto no producían beneficio alguno para la sociedad. Los que estaban fuera de forma y los desempleados era enemigos paradigmáticos que contrastaban con el individuo en forma, representante de la autosuficiencia y autonomía, asociado al éxito y a la autoestima. Este exacerbado sentido del cuidado de uno mismo casaba perfectamente con la cultura de consumo privada que permitía la elección individual de un estilo de vida saludable, la marca definitiva de una vida feliz. Si el fitness yuppie representaba los ideales del nuevo conservadurismo neoliberal, el running se convertía en una herramienta para alcanzar ese modelo y dejaba de ser un camino de autoconocimiento y perfección. Ese cambio de valores puede observarse, por ejemplo, en las campañas de publicidad que aparecían en la revista Runner's World de esta década. Un anuncio de las zapatillas Asics afirmaba:

La GT III. No para las masas. Algunos productores prometen una revolución en las zapatillas, pero acaban dando servicio a un rebaño de conformistas. La nueva Asics GT III es para el corredor en el camino menos recorrido: el camino del líder (Runner's World, junio 1988: 13).

Un ejemplo paradigmático de los valores conservadores de la la Nueva Derecha se veía perfectamente reflejado en los anuncios que Nike introdujo en Runner's World durante 1988, en los que aparece la figura caricaturizada de Ronald Reagan haciendo el saludo militar, motivando la consciencia nacional de los corredores. El titular del anuncio rezaba: «Él no puede correr en el 88. Pero tú sí puedes» y el texto seguía: «La constitución no permite al presidente presentarse de nuevo ${ }^{6}$. Pero nada te impide a ti correr. Porque este año hay más carreras que nunca» (Runner's World febrero, 1988: 71).

Sin embargo, vemos cómo el diseño de las zapatillas de correr no rechazaba de forma total los valores de la etapa anterior. Durante esta etapa reformalizadora se mezclaban valores tales como la flexibilidad y la libertad con nociones de

\footnotetext{
${ }^{6}$ En el texto original en inglés se juega con la expresión «running again» que hemos traducido como "presentarse de nuevo" pero que pierde el doble sentido buscado por los publicistas.
} 
estabilidad, control y confort. Un anuncio de zapatillas de correr Turntech publicitaba su nuevo modelo de sostén anatómico para la estabilidad de talón afirmando:

Los corredores no son cuadrados. Si ves el modo en el que la mayoría de las compañías diseñan zapatillas de correr, pensarías que los corredores tienen pies cuadrados. Si eso fuera cierto, podrían correr en cajas de zapatillas. Pero lo cierto es que los corredores no son cuadrados ${ }^{7}$ (Runner's World, agosto 1987: 5).

Como decía un anuncio de Converse de la época en el que se mostraba un diseño curvo de la suela de la zapatilla: «Nuestra nueva serie AB no rompe las reglas. Solo las tuerce un poco» (Runner's World, abril 1988: 53). Esos valores no quedaban restringidos a los hombres, sino que también se aplicaban a las mujeres. Un anuncio de Nike en el que aparece una habitación sobria con una mujer vestida con ropas de época de los primeros peregrinos que mira a un par de zapatillas Nike azules mostraba el eslogan: «Para mujeres que quieren ponerse en pie de nuevo». El texto continuaba:

Si quieres evitar lesiones, recuerda esto: las mujeres no pronan igual que los hombres. Debido a su estructura de caderas, pronan más. La Nike Air Control es la primera zapatilla de estabilidad diseñada para la forma en que la mujer está construida y cómo se mueve. Con un diseńo anatómico. Extra flexibilidad. Y la mejor amortiguación para cada sexo: Nike Air: la revolución en marcha (Runner's World, agosto 1987: 48-49).

Como observamos, el anuncio mezcla a la vez la rigidez y el control sobre la anatomía del cuerpo de la mujer con ideas de flexibilidad, amortiguación e incluso revolución.

\section{LA REFORMALIZACIÓN TARDÍA DE LA DÉCADA DE 1990}

Si la década de 1980 representaba una fase de reformalización temprana, la década de 1990 suponía una fase de reformalización tardía, conectada con la emergencia de unas nuevas clases medias postindustriales vinculadas a la meritocracia de la sociedad de la información. La clase que David Brooks (2000) denominó como bobos (bohemios burgueses) ejemplificaba la perfecta mezcla entre el espíritu rebelde de los 60 y la fiebre financiera de los 80 . Se convirtieron en la síntesis perfecta entre las fases informalizadoras y reformalizadoras previas. Los bobos conseguían unir paradójicamente el éxito con un cierto espíritu de insumisión. Para ellos, el modo de ganar dinero lo era todo: el dinero debía provenir de una actividad expresiva y no ser utilizado como medio para el consumo y el ocio ostentoso. El dinero debía gas-

7 Otra vez en la traducción se pierde el doble sentido del término «square», que, aparte de la traducción literal de "cuadrado», durante la época de la contracultura de décadas anteriores hacía mención a individuos rígidos, apegados a la costumbre. 
tarse en herramientas necesarias, en lo íntimo y pequeño, en los detalles de la moda retro-chic. La sobriedad era fundamental, algo a expresar en cualquier manifestación, incluso en lo político, donde lo políticamente correcto y un desplazamiento hacia posiciones centristas ganó predominancia. El calculado hedonismo del estilo de vida bobo permitía un control blando por parte de la institución médica en lo que se refería al cuidado de sí desde el punto de vista de la salud y la vida sexual. Los placeres productivos se entendían como buenos y los improductivos como malos. Además, en su relación con la naturaleza, los bobos imaginaban el escapismo de paisajes idílicos que podían ser experimentados de forma lúdica mediante actividades de aventura para intrépidos urbanitas.

La década de los 90, en especial su segunda mitad, vivió la segunda explosión de la carrera (por ejemplo la Maratón de Nueva York contó con 31000 participantes en 1999). En la cultura de la carrera se produjo una mezcla de valores debidos a la revolución cultural de los 60-70 con la cultura de consumo de los 80 . Se reeditaron libros clásicos como el de Sheehan Running and Being, el de Lydiard y Gilmour Running with Lydiard y se recuperaron figuras míticas como la de Bill Bowerman, que sería la imagen de Nike desde el 2000 con el lema «De corredores para corredores». La vuelta a lo subjetivo, lo holístico, al placer de las actividades cotidianas y la autenticidad de las experiencias se introdujeron en una elaborada cultura de consumo a través del running como estilo de vida. Esta síntesis dio pie a una actividad de carrera para todo el mundo. El estilo de vida del corredor se caracterizaba por la nostálgica recuperación de la autenticidad, libertad y naturaleza dentro del marco de un ecologismo responsable. En un artículo llamado «Los 10 mandamientos del correr sano", Bob Wischia enfatiza los sentimientos placenteros, divertidos y agradables de la carrera en vez de programas de entrenamiento rígidos:

En vez de verlo como hacer ejercicio, yo jugaría. Siempre he tratado de considerar mi correr como algo divertido [...]. No soy más rápido o más fuerte, no me fijo marcas personales o alcanzar metas. Simplemente soy más féliz y más saludable (Runner's World, julio 1993: 54).

Todos los cambios de valores que hemos seńalado pueden observarse en los anuncios de las zapatillas que aparecían en la revista Runner's World en los 90. Imágenes de corredores rodeados de paisajes naturales en vez de urbanos se convirtieron en improntas comunes. Como expresaba un anuncio de una sandalia de correr de Diadora: «Ahhh, claros cielos azules, el sol iluminando y la sensación del viento pasando entre mis dedos» (Runner's World, abril 1994: 57). La idea de la aventura en la naturaleza aparecía también en novedosos reportajes sobre carreras de larga distancia. Por ejemplo, en un artículo de Runner's World, Marty Dugard describía la carrera de Resistencia del Golfo de Oman con el mensaje: «¿Quieres aventura? ¿Quieres poner a prueba tu fuerza, resistencia y capacidad? ¿Quieres diez días de experiencias próximas a la muerte en uno de los ambientes más exóticos del mundo? Lo tienes» (septiembre 1993: 65).

Los libros de John Bingham (1999, 2002), considerado en esa década por la revista Runner's World como el nuevo trovador de la carrera, proveen una buena oportunidad para analizar esos valores asociados a la actividad en los 90. Sus obras 
están escritas de forma autobiográfica, trufadas con experiencias personales, sensaciones y sentimientos. El correr aparece como algo de valor para encontrarse a uno mismo, algo a lo que agarrarse como forma de orientación vital. Bigham define la categoría de corredor (runner) de forma genérica, si bien la distingue de la de atleta, de consideración más elevada. Si el corredor es alguien que realiza la actividad de modo informal, el atleta es alguien que corre carreras y que toma la carrera como eje central de su vida. Sin embargo, la competición de las carreras no se enfoca tanto a la mentalidad de ganadores y perdedores sino más bien al autoconocimiento y desarrollo personal. El viaje para convertirse en un atleta no puede ser doloroso, sino que se va desplegando bajo las directrices de un calculado hedonismo porque, como afirma Bingham: «Entrenar duro no era un trabajo, jera divertido!» (1999: 196). Al contrario que la mentalidad "sin dolor no hay mejora» (no pain no gain), el dolor debe ayudarte a saber cuándo parar para poder preservarte para futuras actividades. La parte lúdica e infantil cobra más importancia. Como afirma Bingham: «La mayor revelación fue darme cuenta de que no puedo vivir todos los momentos de mi vida como un adulto. No es necesariamente mejor actuar según tu edad. Yo no lo hago» (Bingham, 1999: 194). Los libros de Bingham atacan un tipo de consumo conspicuo vacío y el afán hipercompetitivo. Eso no quita para que el autor abogue por la compra de productos especializados de calidad y elevado precio:

Sabes que eres un corredor cuando empiezas a utilizar términos como Coolmax, Dri-line, o Dri-fit en tu día a día. Sabes que eres un corredor enterado cuando ponerte una camiseta de algodón te parece tan apropiado como ponerte las zapatillas cambiadas de pie (Bingham, 2002: 39).

Es decir, Bingham considera que los corredores deben rechazar las muestras artificiales y superfluas de superiorismo. Sin embargo, el consumo es aceptable si viene acompañado de compras informadas, vinculadas a lo funcional, no al mero lujo y la pose.

UNA NUEVA TENDENCIA INFORMALIZADORA HACIA MITAD DE LOS 2000 (2004-2011)

A mediados de los 2000, una nueva fase global de informalización implicó una mayor nivelación a nivel político y económico entre distintas naciones alrededor del mundo, con una pérdida de hegemonía del eje occidental (sobre todo americano) respecto a nuevos países industrializados y exportadores de energía. La nueva economía colaborativa de las .com abría la vía a una nueva forma de relaciones laborales más flexibles. Estas circunstancias no implicaban necesariamente y de por sí una mejora automática de las condiciones laborales dentro de cada país y entre países. Como indicaba Wouters (2007; Wouters y Dunning, 2019), las complejas dinámicas de competición/integración del desarrollo global debidas sobre todo al mercado y no a la política propiciaban a la vez una mayor equiparación entre países a nivel mundial pero también una disminución del peso de ciertos grupos sociales en las relaciones de poder. La nueva distribución globalizada del mercado laboral pro- 
movía una desregulación que se vivía de forma muy diferente dependiendo de la clase social (mayor facilidad para el desarrollo personal de la vida laboral en las clases altas pero mayor precarización de clases medias y bajas) y de los propios países (mayor desprotección y explotación de esas clases medias y bajas en los denominados países del tercer mundo) ${ }^{8}$.

En el ámbito de la carrera, apreciamos la llegada de esa fase con la aparición de libros de autores como Romanov (2002), Dreyer (2004) o Mierke (2005), que hacían hincapié en las formas naturales de la carrera. También se dieron estudios científicos sobre la emulación de culturas nativas con técnicas de carrera más acordes con esos patrones naturales de la especie humana. Por ejemplo, Bramble y Lieberman (2004) publicaron en la revista Nature un estudio sobre la carrera de larga distancia en el Homo sapiens. Precisamente en 2004 Nike sacó la zapatilla Nike Free 5.0. En el anuncio de la zapatilla aparecían unos keniatas corriendo por la sabana africana con las Nike en sus pies. En 2006 hace su aparición la marca Vibram, buque insignia de lo que se llamará carrera natural o mínima. En 2007 aparece la compañía Newton, que también favorece el diseño de la carrera natural. Sin embargo, sería en 2009, con la llegada del libro de Christopher McDougall (2009) Born to Run: A Hidden Tribe, Superathletes, and the Greatest Race the World Has Never Seen, cuando el nuevo credo de la carrera natural/mínima y el inicio del trail running (carrera de montaña) llegaron a una gran masa de población. El autor había pasado una larga temporada entrenando con los tarahumara (rarámuri en lengua vernácula), una etnia nativa de las altas sierras de Méjico conocida por las increíbles distancias (de más de $100 \mathrm{~km}$ ) que algunos de sus pobladores realizaban como parte de su tradición, no como actividad competitiva. Como comentaba McDougall, la razón por la cual esos superatletas podían correr esas distancias por la montaña era su conexión con formas naturales?. Pero lo más interesante es que el autor conectaba esas formas de correr de los tarahumara con las que practicaban los pioneros de los 70 :

Nacimos para correr. Todos somos corredores, tal y como los tarahumara siempre lo conocieron [...]. Ya en los 70, los maratonianos estadounidenses se parecían mucho a los tarahumara; eran una tribu de marginados que corrían por amor, confiando tan solo en el instinto y un equipo básico. Si le quitas la parte de arriba a la zapatilla de correr de los 70 tienes una sandalia: la antigua Adidas o la Onitsuka

${ }^{8}$ En 2005 Nike realizó un extenso informe sobre las condiciones de trabajo en las 700 fábricas con las que la marca trabajaba en el sureste asiático, reconociendo públicamente los abusos de los que eran víctimas sus trabajadores. A pesar del intento de Nike de distanciarse de forma directa de estas prácticas, no cabe duda de que es imposible entender el crecimiento global de la marca sin el abaratamiento de costes que supone producir esos productos en países con condiciones laborales más precarias.

9 «Ese era el verdadero secreto de los tarahumara: nunca habían olvidado lo que era amar la carrera. Recordaban que el correr era el primer arte humano, nuestra original e inspirada creación. Mucho antes de que garabateáramos pinturas en las cuevas o hiciéramos ritmos en troncos, ya estábamos perfeccionando el arte de combinar la respiración, la mente y los músculos en una fluida autopropulsión sobre terreno agreste» (McDougall 2009: 92). 
Tigers tan solo eran una suela plana y cordones, sin control de movimiento, soporte del arco plantar o amortiguación de talón (McDougall, 2009: 93).

Los estudios del profesor Daniel Liberman en su laboratorio de biología esquelética de Harvard sobre la carrera descalzo, con publicaciones relevantes en Nature (Liberman et al., 2010) proveyeron el soporte académico a esas formas de carrera natural y mínima. De hecho, las publicaciones sobre la carrera descalzo (barefoot running) continuaron en autores como Saxton (2011), y también las que se referían a etnias que habían mantenido formas de correr más primitivas pero muy eficaces, como por ejemplo los keniatas (Finn, 2012).

Las prácticas de carrera natural y minimalista estaban relacionadas con un tipo de estilo de vida más general que se basaba en la frugalidad y el anticonsumismo. Por ejemplo, el blog de Leo Babauta (autor vegano y minimalista) rezaba en 2010 la "Filosofía del barefoot», en la que afirmaba que "para acoger la filosofía barefoot (sin calzado), no necesitas necesariamente correr descalzo. De nuevo, es una metáfora sobre cómo debes vivir tu vida, y esos principios pueden aplicarse a cualquier cosa que hagas» (Babauta, 2010). A continuación, enunciaba como principios fundamentales de ese estilo de vida la libertad, la ligereza, la desnudez, lo placentero, el estar atento y presente, no ser conformista y no ser consumista, valores que nos recuerdan mucho los encarnados por los corredores de la anterior fase informalizadora de los 70 .

REFORMALIZACIÓN A PRINCIPIOS DE LA DÉCADA DE 2010

No obstante, desde finales de los 2000 y principios de la siguiente década, una nueva ola de reformalización barrió el mundo. En lo económico se produjo una profunda crisis financiera y una transformación de la economía participativa en una industria de la precarización; la mayor desregulación del mercado laboral afectó de manera desigual a los grupos sociales (clase, género, edad, etnia) dentro de los países desarrollados y también a las distintas poblaciones del planeta, generando conflictos de integración y procesos parciales descivilizatorios. En lo político se produjeron movimientos contrarios a una mayor integración: en 2016, la votación favorable al Brexit o la elección de Trump a la presidencia estadounidense constataban la llegada de una nueva perspectiva global en las relaciones geopolíticas y una vuelta al conservadurismo en lo social (centrado en posiciones generales antiinmigración).

En el mundo de la carrera se dejó sentir también cierta reformalización. Como punto de inflexión podríamos considerar lo que ocurrió en 2012, cuando Valerie Bezdek llevó a los tribunales una demanda conjunta (con más de 150000 demandantes) contra la conocida marca de zapatillas de carrera mínima Vibram por publicidad falsa, lo que implicó una pérdida millonaria de la compañía en compensaciones. No obstante, como hemos indicado anteriormente, la fase de reformalización no implica un rechazo pleno y frontal contra los valores y prácticas de la anterior fase informalizadora; tan solo una consolidación y reformulación de lo anterior. Es así, por ejemplo, cómo Nike, que había sido criticada duramente por McDougall como una de las mayores responsables de arruinar la carrera natural debido a 
la densa amortiguación de sus diseños, incluía en 2013 en el diseño de la Free Flyknit una clara referencia a la carrera natural de los rarámuri. El gerente de running de Nike Méjico Luis Godinez afirmaba:

En línea con la nueva filosofía de Nike en la cual se prioriza lo natural, creemos que los rarámuris son una gran fuente de inspiración porque entienden al running como algo natural en sus vidas, como una fuente inagotable de bienestar y disfrute (Nike News, 2013).

Y sobre el propio diseño de la zapatilla se dice: «La Nike Free Flyknit une las dos tecnologías más populares e innovativas para conseguir una compresión que mantiene el pie en su sitio, al tiempo que permite la libertad y flexibilidad de la carrera descalzo" (Nike News, 2013).

Nike conseguía así mantener la idea y valores de lo natural y la libertad de correr asociado a sus zapatillas, algo que continuó a lo largo del tiempo, mezclándolo con nuevos diseños de alta tecnología. Por ejemplo, en 2018 lanzaba las Nike Huarache, término referido a las sandalias de los rarámuri, presentando unas zapatillas ligeras pero que introducían amortiguación y nuevos materiales ${ }^{10}$.

El ejemplo de Nike ejemplificaba bien este período de reformalización en el cual la cultura del running (término que en esta fase se expandió sin traducción más allá de los países anglófonos) se había hecho global y en el cual, como ocurría en la anterior fase de reformalización de los años 90, las ideas de libertad y vuelta a la naturaleza se incluían dentro de un estilo de vida runner como parte de la cultura de consumo. La proliferación de carreras organizadas por ayuntamientos y grandes empresas venía a sustituir el modelo tradicional de carreras populares en las que participaban aficionados al atletismo y expandían la práctica del running a una gran masa de la población, expandiéndose con mucha fuerza entre las mujeres. En EE. UU., en el período de 2004 a 2014 se produjo un incremento de alrededor del $70 \%$ de corredores hasta alcanzar los 42 millones (National Sporting Goods Association, 2014). En 2017, el registro de participación en carreras fue de 18,3 millones de inscritos (Running USA, 2018). La encuesta nacional sobre la carrera de 2017 estimaba que había un 63\% de mujeres corredoras frente a un 37\% de hombres (Running USA, 2017: 9). Además, el 62\% del total se definía como corredores frecuentes/fitness (Running USA, 2017: 11), entre los cuales la motivación principal para correr era el estar sano (77\%) pero en cuarto lugar encontramos el prepararse para una carrera (62\%) (Running USA, 2017: 13). En nuestro país, en la encuesta sobre hábitos deportivos de los españoles de 2015, el porcentaje de personas practicantes de deporte que decían haber corrido el último año era del $30 \%$ (MEC, 2015: 7) mientras que en 2010 el porcentaje era del 12,9\% y en 2005 del 11,1\% (García Ferrando y Llopis, 2011: 87); el porcentaje de población total (entre

${ }^{10}$ Algunas marcas menores como Xero mantuvieron el diseño mínimo de las sandalias de carrera rarámuri. Otras como FBR recibieron gran influencia de la carrera natural de los keniatas para el diseño de las zapatillas. 
15 y 75 años) que practicaba de forma semanal la carrera a pie pasó de 4,8\% en 2010 a 10,6\% en 2015 (MEC, 2015: 92), lo que se traducía en estimaciones de unos 3,7 millones de corredores.

Paralelamente, el incremento de tales carreras desde 2010 ha sido exponencial (solo de 2008 a 2014, en España las carreras se duplicaron, llegando a superar las 3300) y los beneficios asociados a su organización también, algo que aprovecharon los ayuntamientos de distintas ciudades para mejorar las arcas públicas. Como ejemplo, si bien el coste de la maratón de Sevilla está en 1,2 millones de euros, se estima en más de siete el gasto de corredores y acompañantes (López, 2016). Según la consultora NPD Group, el running movía en 2013 en nuestro país alrededor de 300 millones y la venta de zapatillas creció del millón en 2009 a más de dos millones en 2013 (De la Cruz, 2016: 43).

Vemos por tanto cómo se ha conseguido aunar conceptos como estilo de vida runner, asociados a la democratización (tenía cabida todo el mundo) y la sencillez de la práctica, con un formato competitivo, dentro de una lógica de beneficios empresariales -de los cuales participaban en gran medida los ayuntamientos de las ciudades (Pascual, 2013) - que incluían un posicionamiento positivo de marca en sectores como la banca, los seguros o la energía ${ }^{11}$.

Por otro lado, es en esta etapa de reformalización cuando aparece con fuerza otro fenómeno asociado a la carrera: las carreras de obstáculos, representadas por las siglas en inglés OCR (Obstacle Course Race). Si bien hay constancia de esas carreras ya en los 80 y 90 con eventos como la Tough Guy inglesa ${ }^{12}$ y algunas de ellas aparecían recogidas en las páginas de Runner's World ${ }^{13}$, las carreras de obstáculos tuvieron su explosión, tras un tímido comienzo en 2009-2010, a partir de 20122013, siendo sus dos organizaciones más exitosas la Spartan Race y la Tough Mudder. Las carreras de obstáculos mostraban la mezcla de la carrera convencional y el entrenamiento funcional (por ejemplo, el crossfit) y abogaban por un nuevo tipo de fitness más muscular. Como se aseguraba en la propia web de Tough Mudder: «La carrera de distancia puede darte un par de pulmones saludables, pero te dejará con una fuerza de tren superior parecida a la Keira Knighley» (Tough Mudder FACT\# 1, en Sailors et al., 2017: 117). En las carreras de obstáculos se trata de ofrecer emociones fuertes dentro un cierto grado de seguridad, algo que Elias y Dunning (1992)

${ }^{11}$ A este respecto, véase el artículo "Así es la apuesta de las empresas por el running», que apareció en Expansión en 2017. https://www.expansion.com/directivos/deporte-negocio/2017/04/22/ 58fa52d8e5fdea57678b46ac.html.

${ }^{12}$ Carrera originada en 1987 por William Wilson, un antiguo miembro del ejercito británico, tras haber presenciado y participado en las primeras ediciones de la martón de Londres a principios de los 80 . La carrera era anunciada como «el evento más seguro y peligroso del mundo» (Sailors, Teetzel y Weaving, 2017: 116) y sería el germen del que surgiría luego la Tough Mudder.

${ }_{13}$ Por ejemplo, en el número de octubre de 1992, aparece un reportaje sobre la Volkslauf (término alemán traducido como «la carrera de la gente»), una carrea de $10 \mathrm{~km}$ por caminos embarrados que los marines estadounidenses hacían todos los años en Tustin (California) y en la que podían participar también civiles. 
definían como uno de los rasgos característicos del ocio y el deporte. Como el propio presidente de la Tough Mudder, Will Dean, afirmaba:

Se supone que da miedo. Pero dar miedo y ser peligroso no es lo mismo. Una montańa rusa da miedo, una película puede dar miedo pero eso no la hace peligrosa. No hay nada peligroso en este evento. Tenemos que dar un reto a la gente y claro que algunos de los obstáculos intimidan pero eso no quiere decir que no son perfectamente seguros (en Keneally, 2016).

Así mismo, el fundador y presidente de la Spartan Race, Joe de Sena, afirmaba en una entrevista: «Te vamos a poner en una zona de guerra en la que no vas a estar cómodo, sino tan fuera de tu zona de confort que vas a ser más feliz» (London Real, 2015). En esa "zona de guerra amigable» lo fundamental es ser capaz de conectar con el lado más primitivo de nosotros mismos (primera naturaleza), pero manteniendo ciertos controles emocionales (segunda naturaleza), superando los miedos a través de los retos de una carrera exigente. Ese era sin duda uno de los rasgos que Wouters (2007) identificaba como característicos del desarrollo del habitus de tercera naturaleza dentro de procesos de informalización. Es decir, durante esta fase de reformalización en las carreras de obstáculos se consolidaban rasgos de informalización previos con cierta variación, dentro de formatos convencionales y de práctica masiva, algo que se ha extendido internacionalmente (e.g., en Europa encontramos la Strong Viking). En esta fase de reformalización la carrera de obstáculos se inclinó hacia modelos competitivos convencionales (más marcado en la Spartan Race) con la organización de campeonatos europeos, americanos, mundiales. La profesionalización del sector se vio favorecida por la entrada de patrocinadores y medios de comunicación vinculados a las pruebas: desde 2013 Reebok es el patrocinador oficial de la Spartan Race, que en 2016 firmó un contrato con la NBC para retransmitir cinco de las carreras de ese año, incluida la Reebok Spartan Race World Championship. No obstante, al igual que en el caso de la carrera natural/ mínima, las carreras de obstáculos no se conciben tan solo como un modo de rendimiento atlético, sino como un estilo de vida vinculado a formas más naturales. Por ejemplo, en el documental sobre carreras de obstáculos The rise of the sufferfests (Keneally, 2016) aparecen continuas referencias a obras que nada tienen que ver con la cuestión de la carrera, sino con formas más simples de vivir/consumir (Wallman, 2015) o de comer y moverse vinculados al estilo de vida paleo (Durant, 2013), que hace mención a las formas de vida nómadas y hábitos alimenticios de los cazadores-recolectores, anteriores a las comunidades agrarias sedentarias. Como afirmaba Joe de Sena, fundador de la Spartan Race: «Relenticemos el consumismo, empecemos a comer más sano, a sudar más» (London Real, 2015) ${ }^{14}$.

${ }^{14}$ La propia dieta Spartan Race propone un plan de comida cruda para atletas, con los siguientes puntos: «Spartan Tier 1: Suma restando (Omite toda comida procesada); Spartan Tier 2: Pierde tus vicios Spartan Tier 3: Mira más verde; Spartan Tier 4: Libera los animales; Spartan Tier 5: Se tan crudo como sea posible». (https://life.spartan.com/post/raw-diet-for-athletes). 


\section{CONCLUSIONES}

Este artículo ha utilizado la teoría de la informalización de Cas Wouters para llevar a cabo un análisis sobre el desarrollo de la actividad deportiva del running desde mitad del s. xx en EE. UU. y más recientemente como fenómeno deportivo global.

El desarrollo del running se identifica con una tendencia informalizadora en espiral, pasando por diversas fases de informalización y reformalización (tabla 1). La integración de diversos códigos provenientes de diversos grupos sociales de clase, género y etnia pudo identificarse con la variación de los valores y discursos asociados a la práctica en distintos momentos del desarrollo del running.

TABLA 1. FASES DE INFORMALIZACIÓN-REFORMALIZACIÓN EN EL DESARROLLO DE LA CARRERA CON PRINCIPALES CARACTERÍSTICAS Y VALORES ASOCIADOS

\begin{tabular}{|c|c|}
\hline $\begin{array}{l}\text { FASES INFORMALIZACIÓN- } \\
\text { REFORMALIZACIÓN }\end{array}$ & CARACTERÍSTICAS Y VALORES PREDOMINANTES \\
\hline $\begin{array}{l}1960 \text { formalización / } \\
1970 \text { informalización }\end{array}$ & $\begin{array}{l}\text { Carrera con enfoque antiinstitucional, naturalista y con nociones holís- } \\
\text { ticas vinculadas a la salud. }\end{array}$ \\
\hline 1980 Reformalización temprana & Flexibilidad y libertad se mezclan con nociones de control y estabilidad. \\
\hline 1990 Reformalización tardía & $\begin{array}{l}\text { Estilo de vida caracterizado por la recuperación nostálgica de la autenti- } \\
\text { cidad, libertad y naturaleza. }\end{array}$ \\
\hline $\begin{array}{l}2004 \text { Tendencia informalizadora } \\
\text { en ascenso }\end{array}$ & $\begin{array}{l}\text { Carrera natural/mínima. } \\
\text { Anhelo de formas más naturales de carrera. } \\
\text { Identificación con "pueblos nativos» en vías de desarrollo como los } \\
\text { tarahumara mejicanos o los keniatas. }\end{array}$ \\
\hline $\begin{array}{l}\text { 2012/13 Reformalización gana } \\
\text { predominancia }\end{array}$ & $\begin{array}{l}\text { Anhelo de formas naturales/divertidas de correr/fitness. } \\
\text { Estilo de vida runner dentro de cultura de consumo. } \\
\text { Proliferación masiva de carreras populares comerciales. } \\
\text { Explosión de las OCR. } \\
\text { Competición más formal (élite, superiorismo). } \\
\text { Identificación con guerreros (espartanos). }\end{array}$ \\
\hline
\end{tabular}

Si durante los años 60 la tendencia formalizadora mantuvo cierta predominancia, durante la siguiente década la tendencia informalizadora predominó, manifestando un enfoque antiinstitucional, naturalista y de nociones holísticas vinculadas a la salud. Durante los años 80 , una fase de reformalización temprana permitió la mezcla de valores contraculturales de la pasada década con la ética neoliberal de la responsabilidad individual: flexibilidad y libertad se mezclaron con nociones de control y estabilidad. En los años 90 esa fase reformalizadora continuó, dando paso a un estilo de vida vinculado al running, caracterizado por la recuperación nostálgica de la autenticidad, libertad y naturaleza. A mediados de los años 2000 despegó una nueva fase de informalización global en la que prácticas como la carrera natural, correr descalzo o con protección mínima y las carreras de montaña encontraron cierta prominencia. Desde 2012-2013 ganó predominancia una fase de reformalización en la cual el estilo de vida runner se expandió mediante la cultura de consumo y proliferó la organización masiva de carreras populares con fines comer- 
ciales. Además, durante esta fase encontramos el despegue de las carreras de obstáculos (Tough Mudder, Spartan Race), que añadían una versión de fitness más muscular y aportaban formatos organizativos más convencionales.

Tanto la carrera mínima o descalzo como las carreras de obstáculos compartían elementos comunes: presentaban una visión romántica, idealizada con otros no occidentales modernos y una vuelta a la naturaleza. En resumen: promovían el desarrollo de una tercera naturaleza, lo que implicaba un esfuerzo más consciente de vuelta atrás hacia la espontaneidad emocional (primera naturaleza) sin perder los controles interiorizados (segunda naturaleza). No obstante, ambos grupos de actividades mostraban además diferencias vinculadas a su distinto desarrollo cronológico: mientras que la carrera mínima/descalzo (que había vivido su auge durante la etapa informalizadora de los 2000) implicaba una identificación con "gentes nativas» como los tarahumara o los keniatas, las carreras de obstáculos (en alza durante la fase de reformalización que empezó a ganar predominancia entre 2012-13) representaban una identificación con guerreros míticos como los espartanos, lo que representaba un enfoque más «duro» a la vuelta a lo natural y una recuperación de formatos competitivos más convencionales, especialmente en el caso de la Spartan Race, con el elemento central de la élite y mentalidad superiorista de ganadores y perdedores.

Recibido: 5 de septiembe de 2019, aceptado: 14 de enero de 2020 


\section{REFERENCIAS}

Babauta, Leo (2010). «The Barefoot philosophy, in bits». https://zenhabits.net/barefoot-philosophy/, último acceso 2 de septiembre de 2019.

Bingham, John (1999). The Courage to Start. New York: Fireside.

Bingham, John (2002). No Need for Speed: A Beginner's Guide to the Joy of Running. Emmaus: Rodale Press.

Bowerman, William J. y Harris, W.E. (1967). Jogging. New York: Grosset \& Dunlap.

Bourdieu, Pierre (1991). El sentido práctico. Madrid. Taurus.

Bramble Dennis, M. y Lieberman, Daniel. E. (2004). «Endurance running and the Evolution of Homo». Nature, 432: 345-352.

Brooks, David (2000). Bobos in Paradise. New York: New York Times.

De la Cruz, Luis (2016). Contra el running. Jaén: Piedra Papel Libros.

Durant, John (2013). The Paleo Manifesto: Ancient Wisdom for Lifelong Health.

Dutton, Kenneth R. (1995). The Perfectible Body: The Western Ideal of Physical Development. Londres: Kassel.

Elias, Norbert y Dunning, Eric (1992). Deporte y Ocio en el proceso de la civilización. Madrid: FCE.

Elias, Norbert (1995) [1939]. El proceso de la Civilización. Méjico: Fondo de Cultura Económica.

Elias, Norbert (1996). The Germans. Cambridge: Polity Press.

Featherstone, Michael (1987). «Leisure, Symbolic Power and the Life Course», en J. Horne y A. Tomlinson (eds.), Sport, Leisure and Social Relations (pp. 113-138). Londres: Routledge.

FInN, Adharanand (2012). Running with the Kenyans. Croydon: Faber and Faber.

FIxx, James F. (1977). The complete book of running. New York: Random House.

García Ferrando, Manuel y Llopis, Ramón (2011). Encuesta sobre los hábitos deportivos en España 2010. Madrid: CSD.

Henderson, J. (1983). «The Future of Running», en Runner's World Eds., New Guide to Distance Running (pp. 346-356). Mountain View: World Publications.

Howell, Jeremy (1991). «A Revolution in Motion: Advertising and the Politics of Nostalgia». Sociology of Sport Journal, 8 (3): 258-271.

Ingham, A.G. (1985). «From Public Issue to Personal Trouble: Well Being and the Fiscal Crisis of the State». Sociology of Sport Journal, 2 (1): 43-55.

Keneally, Scott (2016). Rise of the Sufferfests. Echo Entertainment.

Kirk, David y Colquhoun, Derek (1989). «Healthism and Physical Education». British Journal of Sociology of Education, 10 (1): 417-434.

Lieberman, D.E., Venkadesan, M., Werbel, W.A., Daoud, A.I., D’andrea, S., Davis, I.S., Mang'Eni, R.O. and Pitsiladis, Y. (2010). «Foot strike patterns and collision forces in habitually barefoot versus shod runner». Nature, 463(7280): 531.

López, David (2016). «El gran negocio de las carreras populares: dónde va a parar el dinero». El Pais, 15 diciembre. https://elpais.com/elpais/2016/12/13/icon/1481633922_713510.html, último acceso 2 septiembre 2019. 
Loret, Alain (1995). Génération glisse. Dans l'eau, l'air, la neige... La révolution du sport des années fun. Paris: Editions Autrement.

London Real (2015). «Joe de Sena-Spartan Race». https://londonreal.tv/e/joe-de-sena-spartan-race/, último acceso 2 septiembre de 2019.

McDougall, Christopher (2009). Born to Run: A Hidden Tribe, Superathletes, and the Greatest Race the World Has Never Seen. New York: Vintage.

MEC (2015). Encuesta de Hábitos deportivos en España 2015. Síntesis de resultados. http://www.culturaydeporte.gob.es/dam/jcr:cd14bedb-feb2-49ea-a433-2565a48d5898/encuesta-de-habitos-deportivos-2015-sintesis-de-resultados.pdf, último acceso 2 de septiembre de 2019.

Midol, Nancy (1993). "Cultural dissents and technical innovations in the 'whiz' sports. International Review for the Sociology of Sport, 28(1): 23-32.

National Sporting Goods Association (2014). Sports Participation in the United States 2014 Edition. Mount Prospect: NSGA.

Nike News (2013). «Nike Free Flyknit Launches in México to Connect with Natural Running». https://news.nike.com/news/nike-free-flyknit-launches-in-mexico-to-connect-with-natural-running, último acceso 2 de septiembre de 2019.

PAscual, Rafael (2013). «El 'running' bate records». Capital, septiembre: 48-50.

Pociello, Christian. (1995). Les cultures sportives. Paris: PUF.

Sailors, P.R., Teetzel, S. y Weaving, C. (2017). Croosfit, Tough Mudder and Spartan.

Rinehart, Robert E. (2000). «Emerging Arriving Sport: Alternatives to Formal Sports», en J. Coakley y E. Dunning (eds.), Handbook of Sports Studies (pp. 504-520). Londres: Sage.

RunNing USA (2017). «2017 National Runner Survey». https://www.runningusa.org/, último acceso 2 septiembre de 2019.

Running USA (2018). «U.S. Road Race Participation Numbers Hold Steady for 2017». https:// runningusa.org/RUSA/News/2018/U.S._Road_Race_Participation_Numbers_Hold_ Steady_for_2017.aspx, último acceso 2 septiembre de 2019.

Sailors, Pam R., Teetzel, Sarah y Weaving, Charlene (2017). «Croosfit, Tough Mudder and Spartan Race», en S.E. Klein (ed.). Defining Sport: Conceptions and Borderlines (pp. 113127). Washington: Lexington Books.

SÁnchez García, Raúl (2008). «Análisis etnometodológico sobre el dinamismo del habitus en Bourdieu y Elias dentro del desarrollo de actividades corporales». Revista Española de Investigaciones Sociológicas (Reis), 124(1): 209-231.

SÁNCHEz García, Raúl (2017). «Civilizing-decivilizing and formalizing-informalizing balances in figurational sociology: an example from the long term development of Japanese martial arts», en A. Ohira (ed.). Civilization, Culture and Knowledge in Process (pp. 51-72). Tokyo: DTP.

SÁNCHez García, Raúl (2018a). «Revisiting duelling and fencing in the sociology of Norbert Elias», en J. Haut, D. Reicher, R. Sánchez-García, y P. Dolan, Exciting Processes-Elias' unpublished papers on Sports, Arts and Body-Cultures (pp. 255-276). Wiesbaden: Springer.

SÁnchez García, Raúl (2018b). «The Development of Mixed Martial Arts: Using the Quest for Excitement and Informalization to Understand Sportization", en D. Malcom y P. Velijja (eds.), Figurational research in Sport, Leisure and Health. London: Routledge.

Sánchez García, Raúl (2018c). The Historical Sociology of Japanese Martial Arts. Londres: Routledge. 
Sánchez García, Raúl y Malcolm, Dominic (2010). «Decivilizing, civilizing or informalizing? The international development of Mixed Martial Arts». International Review for the Sociology of Sport, 45(1): 39-58.

SAxton, Ken B. (2011). Barefoot running. Step by step. Berverly: Fair Winds Press.

Wheaton, Belinda (ed.) (2004). Understanding lifestyle sport: Consumption, identity and difference. Londres: Routledge.

Wouters, Cas (1976). «Is het civilisatieproces van richting veranderd». Amsterdams Sociologisch Tijdschrift, 3(3): 336-360.

Wouters, Cas (1986). «Formalization and informalization: Changing tension balance in civilizing processes». Theory, Culture and Society, 3(2): 1-18.

Wouters, Cas (1999). «Changing patterns of social controls and self-controls. On the rise of crime since the 1950s and the sociogenesis of a 'third nature'». British Journal of Criminology, 39(3): 416-432.

Wouters, Cas (2004). Sex and manners: Female emancipation in the West 1890-2000. Londres: Sage.

Wouters, Cas (2007). Informalization: Manners and emotions since 1890. Londres: Sage.

Wouters, Cas y Dunning, Michael (eds.) (2019). Civilisation and Informalisation: Connecting LongTerm Social and Psychic Processes. Londres: Palgrave. 